\title{
3D models related to the publication: Morphogenesis of the inner ear at different stages of normal human development
}

\author{
TOYODA $S^{1}$, SHIRAKI N ${ }^{1}$, YAMADA $S^{1,2}$, UWABE $C^{2}$, IMAI $\mathbf{H}^{3}$, MATSUDA $\mathrm{T}^{3}$, YONEYAMA ${ }^{4}$, \\ TAKEDA $T^{4}$, TAKAKUWA $T^{{ }^{*}}$
}

${ }^{1}$ Human Health Science, Graduate School of Medicine, Kyoto University, Kyoto 606-8507, Japan

${ }^{2}$ Congenital Anomaly Research Center, Graduate School of Medicine, Kyoto University, Kyoto 606-8501, Japan

${ }^{3}$ Department of Systems Science, Graduate School of Informatics, Kyoto University, Kyoto 606-8507, Japan

${ }^{4}$ Allied Health Science, Kitazato University, Kanagawa 252-0373, Japan

* corresponding author: Dr. Tetsuya Takakuwa (tez@hs.med.kyoto-u.ac.jp)

Abstract: The present 3D Dataset contains the 3D models analyzed in: Toyoda S et al., 2015. Morphogenesis of the inner ear at different stages of normal human development. The Anatomical Record. doi : 10.1002/ar.23268.

Key words: human inner ear, human embryo, magnetic resonance imaging, phase-contrast

Submitted 14.10.2015, Accepted 14.10.2015. doi: 10.18563/m3.1.3.e6

(C) Copyright Tetsuya Takakuwa 2015

\section{SPECIMEN LIST}

The morphogenesis of the human inner ear membranous labyrinth was visualized using images derived from human embryo specimens between Carnegie stage (CS) 17 and post embryonic phase from the Kyoto Collection, which were acquired with a phase-contrast X-ray CT (PCXT) and a magnetic resonance (MR) microscope.

\begin{tabular}{|c|c|c|}
\hline \multicolumn{1}{|c|}{ Specimen ids } & Species & Developmental stage (Crown Rump Length) \\
\hline M3\#36_KC-CS17IER29248 & Homo sapiens & Carnegie stage $17(07.0 \mathrm{~mm})$ \\
\hline M3\#37_KC-CS18IER17746 & Homo sapiens & Carnegie stage $18(12.0 \mathrm{~mm})$ \\
\hline M3\#38_KC-CS19IER16127 & Homo sapiens & Carnegie stage $19(13.0 \mathrm{~mm})$ \\
\hline M3\#39_KC-CS20IER20268 & Homo sapiens & Carnegie stage $20(13.7 \mathrm{~mm})$ \\
\hline M3\#40_KC-CS21IER28066 & Homo sapiens & Carnegie stage $21(16.7 \mathrm{~mm})$ \\
\hline M3\#41_KC-CS22IER35233 & Homo sapiens & Carnegie stage $22(22.0 \mathrm{~mm})$ \\
\hline \hline M3\#42_KC-CS23IER15919 & Homo sapiens & Carnegie stage $23(32.3 \mathrm{~mm})$ \\
\hline \hline M3\#43_KC-FIER52730 & Homo sapiens & Post embryonic phase $(43.5 \mathrm{~mm})$ \\
\hline
\end{tabular}

\section{METHODS}

Well-preserved human embryos between Carnegie stage (CS) 17 and the postembryonic phase during trimester 1 (approximately 6-10 weeks after fertilization) were selected from Kyoto Collection for MR microscopic imaging and phase-contrast X-ray CT (Nishimura et al, 1968; Shiota et al, 2007; O'Rahilly \& Müller, 1987).

The 3D PCXT image acquisition conditions are described elsewhere (Yoneyama et al., 2011). Briefly, specimens were visualized with a phase-contrast imaging system fitted with a crystal X-ray interferometer. The system was set up at the vertical wiggler beam line (PF BL14C) of the Photon Factory in Tsukuba, Japan.

MR images were acquired using a 7T MR system (BioSpec 70/20 USR; Bruker Biospin MRI GmbH; Ettlingen, Germany) with a 35 -mm-diameter $1 \mathrm{H}$ quadrature transmit-receive volume coil (T9988; Bruker Biospin MRI GmbH).

PCXT and MRI data from selected embryos were analyzed precisely as serial $2 \mathrm{D}$ and reconstructed $3 \mathrm{D}$ images. The structure of the inner ear was reconstructed in all samples using Amira software version 5.4.5 (Visage Imaging; Berlin, Germany). The 3D surface models were then processed with ISE-MeshTools (Lebrun, 2014); each model was orientated, tagged and labelled using this software. All tagged surfaces are provided in .vtk format, and labels in .flg format. The 3D surface models are also provided in .ply format, and can therefore be opened with a wider range of freeware.

This study was approved by The Committee of Medical Ethics of Kyoto University Graduate School of Medicine, Kyoto, Japan (E986). 


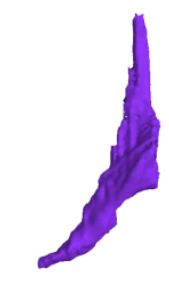

CS17

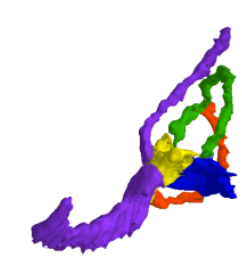

CS19

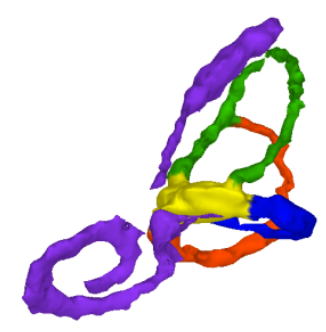

CS22

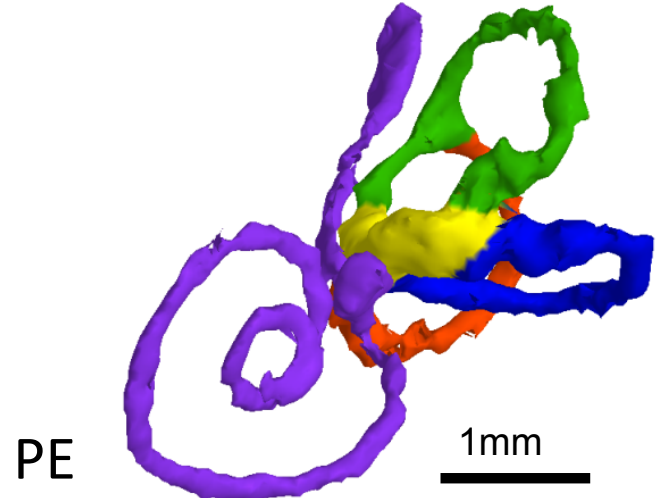

Figure 1: Representative image of the inner ear membranous labyrinth at Carnegie stage (CS)17, CS 19, CS22, and post embryonic phase (PE) (crown-rump length $=43.5 \mathrm{~mm})$. Purple: cochlear duct and lymphatic duct, Yellow: vestibular system, Blue: lateral semicircular duct, Green: anterior semicircular duct and common crus, Red: posterior semicircular duct. scale bar $=1 \mathrm{~mm}$.

\section{ACKNOWLEDGEMENTS}

This study was supported by Grant Nos. 25461642, 24119002, 26220004, 15H01119, 15K08134, 15H05270, 15H01121, and $15 \mathrm{~K} 15014$ from the Japan Society for the Promotion of Science.

This work has been performed under the approval of the Photon Factory Program Advisory Committee (Proposal No. 2013G514, 2012G138, 2014G018, and 2015G574).

\section{BIBLIOGRAPHY}

Lebrun, R., 2014. ISE-MeshTools, a 3D interactive fossil reconstruction freeware. 12th Annual Meeting of EAVP, Torino, Italy.

Toyoda, S., Shiraki, N., Yamada, S., Uwabe, C., Imai, H., Matsuda, T., Yoneyama, A., Takeda, T., Takakuwa, T., 2015 Morphogenesis of the inner ear at different stages of normal human development. Anatomical Record, in press. DOI: 10.1002/ar.23268.
Shiota, K., Yamada, S., Nakatsu-Komatsu, T., Uwabe, C., Kose, K., Matsuda, Y., Haishi, T., Mizuta, S., Matsuda, T., 2007. Visualization of human prenatal development by magnetic resonance imaging (MRI). Am J Med Genet A 143A, 3121-3126. DOI: 10.1002/ajmg.a.31994

Nishimura, H., Takano, K., Tanimura, T., Yasuda, M., 1968. Normal and abnormal development of human embryos: first report of the analysis of 1,213 intact embryos. Teratology 1, 281-290. DOI: 10.1002/tera.1420010306

O’Rahilly, R., Müller, F., 1987. Developmental stages in human embryos: including a revision of Streeter's Horizons and a survey of the Carnegie Collection. Washington, D.C.: Carnegie Institution of Washington.

Yoneyama, A., Yamada, S., Takeda, T., 2011. Fine biomedical imaging using $\mathrm{X}$-ray phase-sensitive technique. In: Gargiulo G, editor. Advanced Biomedical Engineering, Vol. 1. Rijeka: InTech. pp. 107-128. DOI: $\underline{10.5772 / 20456}$ 